\title{
The role of tension-compression asymmetry of the plastic flow on ductility and damage accumulation of porous polycrystals
}

\author{
J. L. Alves ${ }^{\mathrm{a}, *}$, M. C. Oliveira ${ }^{\mathrm{b}}$, L. F. Menezes ${ }^{\mathrm{b}}$, Oana Cazacu ${ }^{\mathrm{c}}$ \\ ${ }^{a}$ MEMS - Microelectromechanical Systems Research Unit, Dep. Mech. Eng., University of Minho, 4800-058 Portugal. \\ ${ }^{b}$ CEMUC, Dep. Mech. Eng., University of Coimbra, Polo II, 3030-788, Portugal. \\ ${ }^{c}$ Dep. Mech. and Aerospace Eng., University of Florida, REEF, FL 32579, USA.
}

\begin{abstract}
The influence of the tension-compression asymmetry of the plastic flow, due to intrinsic single-crystal deformation mechanisms, on porosity evolution and the overall ductility of voided polycrystals is assessed. To this end, detailed micromechanical finite-element analyses of three-dimensional unit cells containing a single initially spherical cavity are carried out. The plastic flow of the matrix (fully-dense material) is described by a criterion that accounts for strength-differential effects induced by deformation twinning of the constituent grains. The dilatational response of porous FCC and BCC polycrystals are calculated for macroscopic proportional tensile loadings corresponding to a fixed value of the stress triaxiality. For the same macroscopic loading, the local plastic strains and the local stress distribution are markedly different from the case when the plastic flow is governed by the von Mises criterion. This has a huge impact on damage accumulation, and ultimately affects the ductility of the porous polycrystals. Most importantly, a direct correlation is established between a macroscopic material parameter that is intimately related to the particularities of the plastic flow and the rate of damage accumulation.
\end{abstract}

(C) 2014 Portuguese Society of Materials (SPM). Published by Elsevier España, S.L. All rights reserved.

Keywords: twinning; porous polycrystals; porosity evolution; ductility

\section{Introduction*}

Beginning with the pioneering studies of Needleman [1972], Tvergaard [1981], Koplik and Needleman [1988], micromechanical finite-element (FE) analyses of unit cells have been used to provide a better understanding of the mechanical response of porous solids. In all these micromechanical studies, it was assumed that the plastic flow of the matrix (void-free material) is governed by the von Mises criterion. However, early on it was recognized that the plastic flow of certain fully-dense, isotropic FCC and BCC polycrystalline materials is not governed by the von

\footnotetext{
* Corresponding author.

E-mail address: jlalves@dem.uminho.pt
}

Mises criterion (e.g. Drucker [1949], Prager and Hodge [1951], Lenhart [1955], Billington [1977]...

In this work a micromechanical study is conducted in order to investigate how the tension-compression asymmetry of the plastic flow, which is due to intrinsic single-crystal plastic deformation mechanisms (e.g. twinning), affects the porosity evolution, and ultimately the ductility of isotropic porous FCC and BCC polycrystals. It is assumed that the porous polycrystal is well described by a 3D periodic array of initially spherical voids surrounded by a fully-dense elastic/plastic matrix displaying tension-compression asymmetry. The plastic flow is described by the isotropic form of Cazacu et al. [2006]'s yield criterion, which is pressure-insensitive, involves all principal values of the stress deviator, and has a single scalar material parameter, $k$, which is intimately related to tension-compression asymmetry. 


\section{Unit FE cell Results and Loadings}

FE computations od a unit FE cell were carried out with DD3IMP [5,6]. Two loading cases were studied: the first one corresponds to the well-known equibiaxial tensile stress state (EBT), a loading of great practical importance in metal forming. The macroscopic measures of the EBT are:

a) $\mathbf{T}_{\Sigma}=+2 / 3$ and $\boldsymbol{\mu}_{\Sigma}=-1$,i.e. $\Sigma_{1} / \Sigma_{2}=1$ and $\Sigma_{3}=0$; the second loading corresponds to the same stress triaxiality but positive Lode parameter, namely:

b) $\mathbf{T}_{\Sigma}=+\mathbf{2} / \mathbf{3}$ and $\boldsymbol{\mu}_{\Sigma}=+\mathbf{1}$,i.e. $\Sigma_{1} / \Sigma_{2}=1$ and $\Sigma_{3} / \Sigma_{1}=4$.

\section{Numerical results}

Results concerning the damage evolution (or evolution of the void volume fraction with the equivalent strain) are shown in Fig. 1 in case of equibiaxial loading $\left(T_{\Sigma}=+2 / 3\right.$ and $\left.\mu_{\Sigma}=-1\right)$, and in Fig. 2 in case of the loading with the same stress triaxiality but positive Lode parameter, i.e. $\mathbf{T}_{\Sigma}=+\mathbf{2} / \mathbf{3}$ and $\boldsymbol{\mu}_{\Sigma}=+\mathbf{1}$.

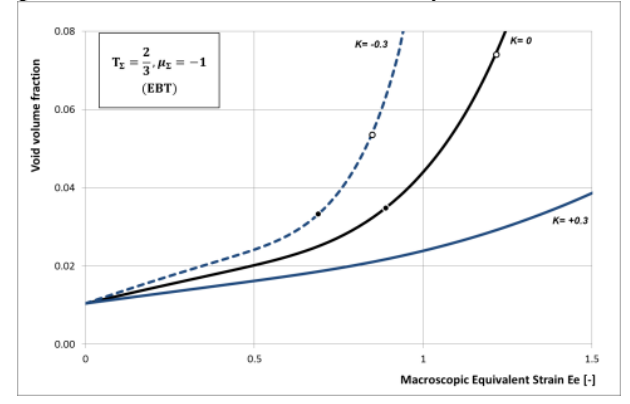

Fig. 1. Evolution of the void volume fraction with the macroscopic equivalent strain $\mathrm{Ee}$, obtained by cell calculations for materials with matrix characterized by: $\mathrm{k}=-0.3$, Mises $(\mathrm{k}=0)$ and $\mathrm{k}=+0.3$.

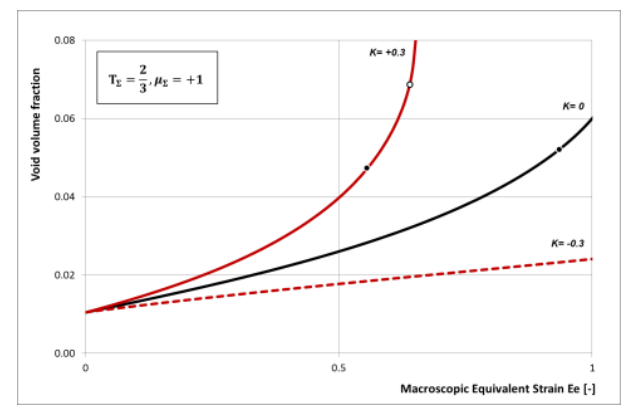

Fig. 2. Evolution of the void volume fraction with the macroscopic equivalent strain Ee, obtained by cell calculations for materials with matrix characterized by: $\mathrm{k}=-0.3$, Mises $(\mathrm{k}=0)$ and $\mathrm{k}=+0.3$.

The most interesting point to be highlighted is how the specificities of the plastic flow of the matrix affect every aspect of the mechanical response of the porous solid, in particular it is clearly shown a strong coupling between the sign of Lode parameter, i.e. the effect of $\mathbf{J}_{3}$, and the ductility and damage accumulation on materials exhibiting different tension-compression asymmetries. In detail, under EBT (i.e. $\mu_{\Sigma}=-1$ ), the FCC polycrystal, which is characterized by $\mathrm{k}=-0.3$ exhibits a much fast void growth than in case of the other materials, while in case of the BCC polycrystal, characterized by $\mathrm{k}=+0.30$, the rate of void growth is the slowest, and thus a much larger ductility can be attained.

\section{Conclusions}

The effect of the tension-compression asymmetry in plastic flow, due to intrinsic single-crystal deformation mechanisms such as twinning, on porosity evolution and on the overall ductility of voided polycrystals was assessed by FE cell calculations.

It was clearly shown that the tension-compression asymmetry in the plastic flow of the polycrystals, described by the parameter $k$ of CPB06 [2], has a very strong influence on all aspects of the mechanical response of the porous polycrystals.

These results point out that optimum ductility cannot be seen without combining the material properties with the deformation process: a "tailored" material can be optimized to perform well for a given deformation process.

\section{Acknowledgements}

The authors gratefully acknowledge the financial support of the Portuguese Foundation for Science and Technology (FCT) via the project PTDC/EMETEC/1805/2012.

\section{References}

[1] Alves, J.L., Revil-Baudard, B., Cazacu, O. (2014) Mod and Sim in Mat Science and Eng, 22(2), 025005.

[2] Cazacu, O., Plunkett, B., Barlat, F. (2006) Int. J. Plasticity. 22, 1171-1194.

[3] Hosford, W.F. and Allen, T.J. (1973) Met. Trans. 4, 1424-1425.

[4] Koplik, J., Needleman, A. (1988) Int. J. Solids Struct. 24 (8), 835-853.

[5] Menezes, L.F., Neto, D.M., Oliveira, M.C., Alves, J.L. (2011) ESAFORM Conf Proc, 1353, 5-10, 1220-1225.

[6] Neto, D.M., Oliveira, M.C., Menezes, L.F., Alves, J.L. (2014), Comput. Method Appl. Mech. Eng. 271, 296-320.

[8] Alves, J.L., Cazacu, O. (2015) European J Mechanics A/Solids, Volume 51, 44-54.

[9] Alves, J.L., Cazacu, O. (2015) Comptes Rendus Mécanique, Vol 343 (2), 107-120. 